\title{
Lupus Enteritis: An Important Cause to Be Considered in Systemic Lupus Erythematosus Patients with Acute Abdomen - A Case Report
}

\author{
Ajit Surin ${ }^{1}$, Aswin Nair ${ }^{2}$, George Jacob ${ }^{3}$ and Debashish Danda ${ }^{4}$ \\ ${ }^{1,2,4}$ Department of Clinical Immunology and Rheumatology, Christian Medical College and Hospital, \\ Vellore, India \\ ${ }^{3}$ Department of Radiodiagnosis, Christian Medical College and Hospital, Vellore, India
}

Correspondence should be addressed to: Ajit Surin; ajitcmc@gmail.com

Received date: 23 September 2013; Accepted date: 30 October 2013; Published date: 20 December 2013

Academic Editor: Carlos Silva Vasconcelos

Copyright @ $\subseteq$ 2014. Ajit Surin, Aswin Nair, George Jacob and Debashish Danda. Distributed under Creative Commons CC-BY 3.0

\begin{abstract}
Lupus Enteritis should be considered in SLE (Systemic lupus erythematosus) patients presenting with acute abdomen. We present a case report of a 15 year old girl who presented to us with acute abdomen. Computed tomography (CT) scan abdomen revealed features of enteritis, which was helpful in avoiding surgery.
\end{abstract}

Keywords: SLE, Lupus Enteritis

\section{Introduction}

Systemic lupus erythematosus (SLE) is a systemic auto-immune disease with multiorgan involvement. The gastrointestinal tract can be commonly affected in SLE, with almost half the patients presenting with related symptoms (1). Most gastrointestinal manifestations are caused by adverse reaction of medicines or infections, while the symptoms related to the disease are not as common as other organ involvement. However, abdominal pain is a frequent symptom in patients with SLE and a challenging diagnostic and therapeutic dilemma as patients may be on steroids and immunosuppression, which may mask the manifestations of ischemia or perforation (2, 3).

\section{Case Report}

A 15 year old girl, with history of vascular headache over the past 1 year was brought to the emergency department with complaints of intermittent fever, persisting headache, easy fatigability and extreme tiredness for 6 months. Over the last 4 months she had

Cite this Article as: Ajit Surin, Aswin Nair, George Jacob and Debashish Danda (2014)," Lupus Enteritis: An Important Cause to Be Considered in Systemic Lupus Erythematosus Patients with Acute Abdomen - A Case Report", International Journal of Case Reports in Medicine, Vol. 2014 (2014), Article ID 608426, 
developed excessive hair loss and hyperpigmented non-pruritic skin lesions over the face, back and ear lobes, without any other specific systemic complaints.Two months before; she reported an acute episode of a spreading abdominal pain with recurrent bouts of vomiting without significant alterations in bowel movements or frequency. She was admitted to the local medical facility where she was initially thought to have sub-acute intestinal obstruction and treated with pain relieving measures.During this admission she was diagnosed as SLE with positive ANA, elevated antibodies to anti-DsDNA and low complements. Subsequently she was initiated on low dose oral steroids and antimalarial. She had transient improvement of her symptoms, with recurrence despite being on treatment.

During examination, she was pale, had discoid lupus lesions over her trunk, face and limbs. She had ulcer over the hard palate. She had frontal hair loss and hyperpigmented macules over the face, trunk, ear lobes and soles. Abdominal examination revealed sluggish bowel sounds. Initial workup revealed anemia, hypoalbuminemia and bland urine examination. Pancreatic enzymes were within normal range. Serological evaluation revealed positive ANA (speckled pattern), with extremely low complement [C3 26.7mg/dl (normal 90 - 180), C4<5.42mg/dl (normal 10-40)], normal antiDsDNA-57 IU/ml (normal<100IU/ml), with positive anti-phospholipid antbodies. Lupus Anticoagulant was mildly positive, anticardiolipin antibodies were $33 \mathrm{AU} / \mathrm{ml}$ (normal < 12 units/ $\mathrm{ml}$ ), IgM antibodies to $\beta 2$ - Glycoprotein-1 $14 \mathrm{IU} / \mathrm{ml}$ (normal < 20
IU / $\mathrm{ml}$ ) and IgG antibodies to $\beta 2$ Glycoprotein-1 302 IU / ml (normal < 20 IU / $\mathrm{ml}$ ). Based on clinical examination and serology reports a diagnosis of SLE with secondary anti-phospholipid syndrome (APS) was considered. Her SLE disease activity index (SLEDAI-2K) score was 17, indicating high disease activity. She was continued on low dose steroids and antimalarial, with a plan to increase immunosuppression based on the organ involvement, after ruling out other causes for fever.

Two days following admission, she developed excruciating abdominal pain associated with loose stools. On examination she had diffuse abdominal tenderness, with guarding and sluggish bowel sounds. Plain radiograph of abdomen was normal. Ultrasound study of the abdomen showed mild hydroureteronephrosis and mild ascites. Differentials of intestinal pseudoobstruction (in view of its association with hydroureteronephrosis), pancreatitis, lupus enteritis and infection were considered). She was started on empirical intravenous antibiotics. An opinion from the GI surgeon was sought, who suggested emergency exploratory laparotomy for evaluation. A CT abdomen as taken prior to the laparotomy, which revealed ileal loop wall thickening with mesenteric fat stranding, abnormal bowel wall enhancement (target sign), mild engorgement of mesenteric vessels, with increased number of visible vessels (comb sign) with no evidence to suggest pancreatitis or obstruction (Figure - 1) . 


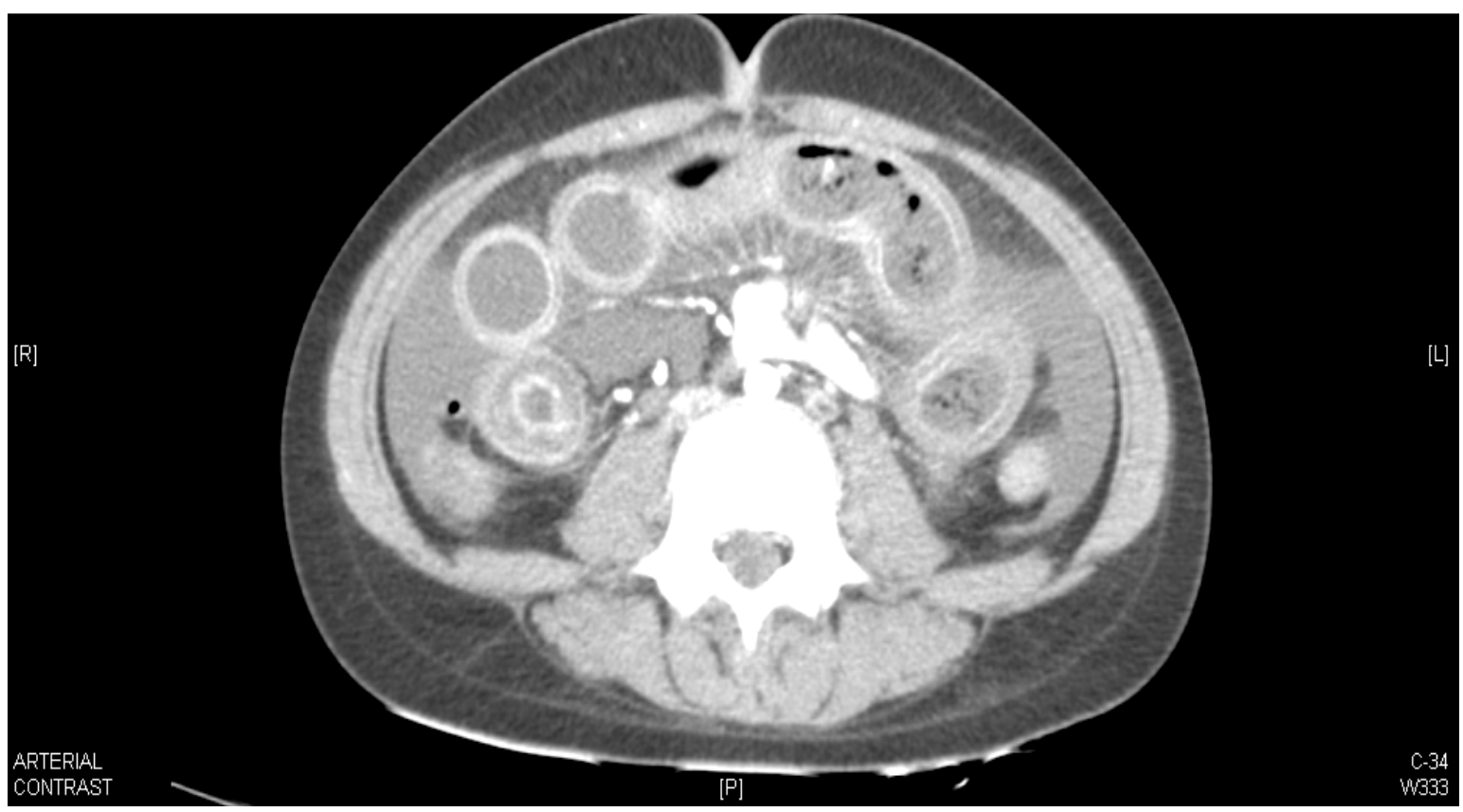

Figure 1: 'CT Abdomen with intravenous contrast demonstrating circumferential thickening of ileal loops with submucosal edema and abnormal enhancement (Target Sign). Mesenteric changes in the form of increased fat attenuation, engorged mesenteric vessels (Comb sign) and ascites also noted.'

Considering the classical imaging findings of lupus enteritis the laparotomy was deferred. She was pulsed with parenteral Methyl Prednisolone (750 mg infusion per day) for 3 days, along with heparin and antibiotics. Following the pulse therapy she showed remarkable improvement, with resolution of the abdominal pain and loose stools. Subsequently Mycophenolate mofetil and oral steroids were started. Heparin was discontinued prior to discharge and Ecospirin was prescribed. Patient was well and symptom free at the time of discharge.

\section{Discussion}

Lupus enteritis, also termed as mesenteric arteritis, lupus mesenteric vasculitis, lupus arteritis, lupus vasculitis, is a potentially severe complication of SLE, stressing the need for swift diagnosis and adequate management. In the BILAG 2004, lupus enteritis is defined as either vasculitis or inflammation of the small bowel, with supportive image and/or biopsy findings, thus a poorly defined cause of abdominal pain in SLE (4).

Mean age at diagnosis of lupus enteritis reported in literature is 32.5 years, with the youngest patient being 13 and the oldest being 72 years old and male-female ratio of $1: 14$ (5). Abdominal pain is the most common presenting symptom amongst other non-specific manifestations like ascites, nausea, vomiting, diarrhea and fever and has been shown to be the most common cause of abdominal pain in SLE patients seeking emergency room treatment (6). Typically, the abdominal pain described in lupus enteritis is diffuse in pattern and in some cases accompanied by rebound tenderness and abdominal muscle guarding (1). Other than a drop in white blood cell count and complement titres, which may correlate with the occurrence of lupus enteritis, none of the laboratory indices have been found to be useful to establish the diagnosis $(5,6)$. The 
patient discussed had Low complement levels. Medina et al (7) and Buck et al (8) have reported that gastrointestinal vasculitis or thrombosis in SLE had higher SLEDAI scores than patients with active SLE with non-SLE related acute abdomen, but no such correlation was established by Lee et al (6). However, the presence of autoantibodies against phospholipids and endothelial cells might provide information about the likelihood of recurrence of lupus mesenteric vasculitis (9).

CT scanning has become the investigation of choice for diagnosis of lupus enteritis (10). Features of lupus enteritis described include focal or diffuse bowel-wall thickening, bowel dilation, target sign, comb sign (considered to be an early sign), increased attenuation of mesenteric fat and ascites. Target Sign is "thickened bowel wall with enhancing outer (muscularis propria/serosa) and inner (mucosa) layers and a hypoenhancing middle layer due to submucosal edema."

Bowel ischemia due to lupus enteritis has been shown to be less ominous than previously expected, and the abnormal CT findings were reversible with early diagnosis and prompt treatment (11). Lack of specificity of these signs is a limitation of CT because they can also be seen in patients with pancreatitis, mechanical bowel obstruction, peritonitis, or inflammatory bowel disease, all of which may mimic intestinal ischemia $(1,5,6)$.

There are no randomized control trials on treatment of this entity. Lupus enteritis has been found in several reports to be generally reversible and steroid responsive, thus considered as the first line therapy $(1,5)$. High dose prednisolone, Methylprednisolone pulse have been used according to individual preferences with favourable responses along with supportive measures which include bowel rest, IV fluids, proton pump inhibitors and Heparin might be added in presence of ApL (Antiphospholipid antibody) antibodies and suspicion of Antiphoshpholipid syndrome $(1,3,5)$. Cyclophosphamide or Mycophenolate may be added in case of resistance to corticosteroids or when warranted by other organ involvement (1). Complete resolution of acute abdominal pain took a few weeks and no patients developed GI perforation in the series by Lee et al., (6). Medina et al., (7) emphasizes the importance of early laparotomy as it influences positively on the prognosis of SLE patients with acute abdomen, however seems unnecessary with the available evidence unless there is a strong suspicion of perforation. A precise diagnosis of lupus enteritis is absolutely essential to commence the patient on appropriate immunosuppressive therapy, thus avoiding a surgical procedure.

\section{Conclusion}

One should think of lupus enteritis in patients of SLE presenting with acute pain abdomen and plain CT abdomen helps to confirm the diagnosis.

\section{References}

1. Buck AC, Serebro LH, Quinet RJ. Subacute abdominal pain requiring hospitalization in a systemic lupus erythematosus patient: a retrospective analysis and review of the literature. Lupus 2001, 10:491-495.

2. Byun JY, Ha HK, Yu SY and Min JK. CT features of systemic lupus erythematosus in patients with acute abdominal pain: emphasis on ischemic bowel disease. Radiology 1999; 211:203-209.

3. Janssens P, Arnaud L, Galicier L. Lupus enteritis: from clinical findings to therapeutic management. Orphanet Journal of Rare Diseases 2013, 8:67.

4. Ju JH, Min JK, Jung CK. Lupus mesenteric vasculitis can cause acute abdominal pain in patients with SLE. Nat Rev Rheumatol.2009; 5:273-81.

5. Ko SF, Lee TY, Cheng TT, Ng SH. CT findings at lupus mesenteric vasculitis. Acta Radiol 1997, 38:115-120. 
6. Kwok SK, Seo SH, Ju JH, Park KS. Lupus enteritis: clinical characteristics, risk factor for relapse and association with antiendothelial cell antibody. Lupus 2007, 16:803-809.

7. Lee CK, Ahn MS, Lee EY. Acute abdominal pain in SLE: focus on lupus enteritis (GI vasculitis). Ann Rheum Dis 2002; 61:54750.

8. Medina F, Ayala A, Jara LJ, Becerra M, Miranda JM, Fraga A. Acute abdomen in systemic lupus erythematosus: the importance of early laparotomy. Am J Med 1997; 103:100-105.
9. Sultan SM, Ioannou Y, Isenberg DA. A review of gastrointestinal manifestations of systemic lupus erythematosus. Rheumatology 1999; 38:917-32.

10.Tian XP, Zhang X. Gastrointestinal involvement in systemic lupus erythematosus: Insight into pathogenesis, diagnosis and treatment. World J Gastroenterol 2010; 16:2971-77.

11. Yee CS, Gordon C, Isenberg DA, Griffiths B. The BILAG-2004 systems tally a novel way of representing the BILAG2004 index scores longitudinally. Rheumatology 2012; 51:2099-105. 